\title{
Normalization Across Species of Laryngeal Physiology
}

\author{
Donald S. Cooper, Ph.D.
}

\begin{abstract}
Much research on structures underlying speech is based on non-human models. Sometimes we wish to know about non-human species, but more often often our basic objective is to apply the information acquired to humans. The objective of this paper is to indicate a strategy for this purpose, and exemplify it in regard to the larynx.

We may depict one particular animal group such as the eutherian mammals in terms of an archetype, from which the traits of constituent groups can be derived. This schema is applied to specify the relations between simultaneously existing species such as the subjects of our experiments.

The way to quantification of such relations was shown by D'Arcy Thompson. An important aspect explored here is the scaling of different species in relation to their spatial dimensions and physiology. We explore such scaling in regard to the size, mass, force, speed of contraction, fatigue-resistance, and precision of control of laryngeal muscles.

The use of appropriate procedures for physiological scaling, and other metaanalytic procedures, can assist us in the integration of available information abont laryngeal physiology, building on data from non-human species to improve the understanding of laryngeal function in humans.
\end{abstract}

Key words : laryngeal physiology, normaligation, laryngeal model, cross-species comparison

\section{Introduction}

Specialists of many sorts inquire abont the function of the structures underlying human speech and voice. However, some sorts of information cannot be obtained directly from human laboratory subjects, or from patients. For this reason, researchers often seek information about the function of homologous structures in non-human species.

Some researchers have argued that nothing relevant can be learned about the function in speech of the structures underlying speech and voice from species that do not speak. This opinion overlooks the fact that the main functions of these structures are in deglutition and respiration, which differ in detail between man and other species, but coincide in most respects.

what attitude should a researcher take in regard to the results of animal experimentation? A careful approach was exprssed by Hast (1969) ${ }^{1}$. At the conclusion of a study in which he compared measures made on laryngeal muscles of dogs and cats with comparable measures on non-human primates, he wrote "The physiological similarity of the dog and the primate larynx substantiates the dog's value for research on the neuromuscular mechanisms of the larynx."This approach has sometimes been replaced by a less cautious one in which concrete

Department of Speech Phathology and Audiology, University of South Carolina : Columbia, South Carolina, 29208 U.S.A. e-mail : doncoop@ mindspring. com

原稿受理：2001 年 1 月 12 日 
results from non-human species are directly substituted for unavailable results from humans. More broadly founded procedures for the cross-species scaling of mammalian physiological processes will be discussed here. They will be exemplified on the neuromuscular physiology of the larynx, but they are applicable to other physiological systems, throughout the mammalian body. The distinctive physiological characteristics of laryngeal muscles to be considered here are their geometry, mass, strength, their speed of contraction, their resistance to fatigue, and the precision of their control.

\section{Evolutionary Versus Archetypal Frameworks}

Now let us return to our question. When we have obtained information from various experimental animals about some aspect of laryngeal function, what can we do with it? what generality does this information have, and how can we apply it to human beings? Ordinarily we think of the relations between mammalian species in an evolutionary context. The perspective adopted here is somewhat different.

The information which underlies evolurtionary studies comes partly from comparative anatomy : in the present case, we join to it information from comparative physiology. But we do not ask, what is the evolutionary descent of given structures and functions? Instead, we ask, can we construct part of an archetype, a conceptual standard mammal, from which we can estimate the function of the larynx in particular cases, once we know such things as the size of the animal and particular adaptations?

This choice of perspective brings to mind echoes of the nineteenth-century discussion of the origin of species. Those who did not recognize evolution, such as the great paleontologist Richard Owen, saw the relations between animal species in terms of archetypes of groups of species. Even T.H.Huxley, later the fiercest advocate of evolution, at first refused to recognize the mutability of species, and considered animal species in this archetypal framework (Desmond ${ }^{2}$, 1993).

Since it is necessary to compare the quantitative results of many investions, a more modern approach has come partly from biostatistics. The biostatis- tician Stahl ${ }^{3}$ (1965) concluded from such studies that "all animals have in common a basic kind of "physiolgical design”. “In a later study ${ }^{4)}(1967)$, he concluded that general design parameters could be obtained which specified the normal mammalian design.

When snch a framework is applied to contemporaneously living animals of the present age, it provides a normative perspective. If we can specify a systematic and general relationship (or several) which accounts for a large part of the cross-species variation of interest, then we can normalize across species. Given the general character of the larynx, factos such as animal mass, the stage of maturation, the sex, and the state of health of each organism will affect the structure and function which will be found in a particular larynx. In this discussion the effect of animal mass and cross species scaling will be explored.

\section{Biological Scaling}

The modern landmark in studies of biological scale is the work of D'Arcy Thompson ${ }^{5}$, a British zoologist who combined his biological studies with formidable competence in mathematics and classical literature. His classic study of biological scaling is embodied in the two editions of his monograph On Growth and Form (1917, 1942). We follow Bonner's revision (1961). Thompson was followed by Julian Huxley $^{6)}$ (1932) partly in collaboration with G. Teissier. Important modern overviews are by McMahon and Bonner ${ }^{7}(1983)$, Peters $(1983)^{8}$, Schmidt-Neilsen ${ }^{9}$ (1984), Calder ${ }^{10)}$ (1984-1996), and Brown and West ${ }^{11)}(2000)$.

\section{The Allometric Formula}

In physiological studies, simple relationships of proportion are commonly embodied in power functions of the form

$$
\mathrm{Y}=\mathbf{a} \times \mathrm{M}^{\mathrm{b}}
$$

Such a relationship is known as an allometric formula, in which the mass of the animal (M, commonly in kilograms) constitutes the argument (independent variable), while the dependent variable $\underline{Y}$ is the physiological quantity which varies as a function of animal mass. The parameter $\underline{a}$ is the value of the 
quantity for an animal with a mass of one kilogram, and the parameter $\underline{b}$ is the slope of the function on a log-log graph (Stahl') 1967). The exponent $\underline{b}$ is most often fractional. In some cases more complex formulations are required. Compare Batschelet ${ }^{12)}$ (1976) with references on the mathematical background.

\section{Size and Proportionality}

Within a given species, the general shape is most often sufficiently constant to use constant proportional scaling, i.e. the exponent $\underline{b}$ changes little across animals of different sizes. In this case, we can compare animals simply on the basis of their mass, which will be proportional to the cube of linear dimensions such as the length of a limb. Following Thompson $^{5}(1961)$, we can justify this if we consider that the volume of a cube will vary as the third power (e.g. $\mathrm{L}^{3}$ where $\mathrm{L}$ is in meters) of the length of a side. The mass can be calculated from knowledge of the average density of the material which composes the cube, as the product of the density and volume of the structure.

The mass of an organism will thus be proportional to, rather than equal to, the third power of the linear dimensions. For instance, in the case of proportional scaling, if a linear dimension such as the length of a limb is doubled, we can expect that the mass of the animal will increase by eight times. Because the mass of an animal is more easily obtained in practice and is more commonly tabulated than informations on its linear dimensions, this formulation allows comparison of dimensions as. long as the proportions of the animal remain constant across the range of magnitude in question.

If for any measurable dimension of the larynx we find that it is not proportional to size, then general anatomical proportionality cannot be assumed. Let us consider one example for which systematic data are available. Harrison ${ }^{13}$ (1995) lists muscle area for the cricothyroid muscle in 62 species. Harrison incorrectly compared the area of the muscle (proportional to the square of linear dimensions) to the animal mass (proportional to the cube of linear dimensions), finding a linear regression which was not significant at the $95 \%$ level. For this reason, the statistics were recalculated with a logarithmic data transformation to produce a power function such as discussed above ; this resulted in a linearized regression with a. 8571 Pearson correlation coefficient highly significant at the $95 \%$ level. The result is that overall anatomic proportionality between the area of the muscle and the animal's mass is preserved for the cricothyroid muscle in this sample extending in size from a $1.33 \mathrm{~kg}$ tree hyrax to a $3800 \mathrm{~kg}$ hippopotamus.

We have given a partial quantitative aspect to the familiar fact that while the general design of the larynx is similar across most mammalian species, there is some anatomical variability in its proportions. However, this is a different question from asking whether we can establish a physiological parallelism across species which holds up despite differences of anatomical detail and scale.

We consider this alternative because the role of the larynx is ultimately metabolic. It is a valve in the system which provides oxygen for oxidative metabolism. Thus there may be physiological constancies or proportions which depend on how much tissue tissue mass mass must be supplied with oxygen, and are maintained despite lack of strict anatomical proportionality.

\section{Non Proportional Scaling}

The calculation above was based on the assumption of proportionnal scaling, or geometric similarity between the animals compared. However, as Galileo noted in 1637, the corresponding structures of animals become heavier and thicker as the overall mass of the animal increases. For this reason, a better fit may be provided not by a model which preserves qeometric proportions, but by an affine transformation, in which the volume of corresponding structures are in the same proportion to the overall volume of the animal in specimens of different size $\left(\mathrm{McMahon}^{14)} 1980\right.$ ). This will produce the sort of systematic distortion of shape with increasing size which in fact is found in many animal groups. For reasons of space, we can only suggest the results of alternative scaling procedures. 


\section{Laryngeal Muscle Mass and Animal Mass}

It has been established that the masses of single muscles are in general linearly proportional to the animal's mass (Calder ${ }^{10}$ 1996). This means that we can predict the mass of a given muscle by an expression of the general form

mass of specific muscle $=\mathrm{a} \times \mathrm{M}^{1.0}$

where body mass $\underline{M}$ is usually in kilograms. The exponent for body mass may vary slightly from unity. The coefficient $\underline{\mathrm{a}}$ and the exponent must be determined separately for each muscle from cases in which the muscle mass has been measured.

For a simple case in which the exponent is 1.0, we can find the coefficient $\underline{\text { a }}$ on the basis of the average available values for muscle mass and body mass :

$$
\mathrm{a}=\text { muscle mass } / \mathrm{M}
$$

For instance, on the basis of Faaborg-Andersen's ${ }^{15)}$ (1957) values for human subject mass and muscle mass, a for the posterior cricoarytenoid (PCA) muscle would be : $9.506 \times 10^{-6}$. Thus, from knowledge of the mass of the animal, we can estimate individual muscle mass. From knowledge of muscle density (about $1.06 \mathrm{~g} / \mathrm{cm}^{3}$ ), it is possible to estimate the muscle volume.

The muscle's length can be estimated in turn on the basis of the ratio of the cube roots (i.e. ratio of linear dimensions) of the masses. Given estimates of the volume and length of the muscle, it is possible to estimate its cross-sectional area.

\section{Animal Size and Isometric Force}

Muscles have a range of mechanical output. One can estimate the maximal tetanic response of the muscle in a given configuration as the product of the muscle cross-sectional area and the maximal force per unit cross-sectional area. Skeletal muscles of animals such as frogs and mammals produce a maximal isometric tetanic tension with a mid-range value of about $28 \mathrm{~g} / \mathrm{mm}^{2}$ (Wilkie ${ }^{16)}, 1976$ ).

The cross-sectional area of a given muscle would be expected to vary as the $2 / 3$ power of animal mass $^{17)}$ (Hill 1950) with proportional scaling, or as $\mathrm{M}^{3 / 4}$ following McMahon ${ }^{14)}$ (1980).

In a study of Hast ${ }^{18)}$ (1966), with dogs of a weight from 10 to $14 \mathrm{kgs}$, and a mean thyroarytenoid (TA) muscle length of abont $16 \mathrm{~mm}$, the isometric tetanic force at resting length was a mean $165 \mathrm{~g}$. Rice and Cooper $^{19)}$ (1989) found an average tetanic force of $66 \mathrm{~g}$ in the unoperated TA muscle of small dogs with an average weight of $6.7 \mathrm{~g}$ and with a muscle length of about $10 \mathrm{~mm}$. Here the TA muscle length was a better predictor (estimated 64g) across species than was animal mass, suggesting imperfect proportionality. Corrections of varius sorts may be necessary. Various studies (e.g. Hast ${ }^{18)}$ 1966) give ratios between the magnitude of twitch and tetanic force, making it possible to esimate twitch force across species.

\section{Physiological Time}

So far, we have considered only spatial dimensions, while physiological processes take place over time. Before considering such time-related indices as contraction time and fatigue-resistance of muscles, it is indispensable to introduce the concept of physiological time.

We tend to be aware that smaller animals live less long than large ones. This relation is quite specific. The average mammalian lifespan (McMahon and Bonner ${ }^{7)}$, 1983) has a duration of $3.3 \times 10^{8}$ breath cycles, and during this time the animal will produce about $1.5 \times 10^{9}$ heartbeats. This is almost independent of the size of the animal. Of course, these events do not have the same duration in absolute temporal terms. The absolute frequency and durations of the respiratory and cardiac cycles can be estimated from the mass of the animal (Stahl), 1967), McMahon ${ }^{14)}$ (1980) has found the duration of specific physiological events to scale approrimately proportionally to the fourth root of the mass of the animal, or $\mathrm{M}^{1 / 4}$. A.V. Hill ${ }^{17)}$ (1950) estimated that animals carry out similar movements and live in times that are proportional to the ratio of their linear dimensions : i.e. physiological time is proportional to $\mathrm{M}^{2 / 3}$.

\section{Contraction Time of Laryngal Muscles}

It should be noted that the statements cited above refer to movement. They would apply to an isotonic type of experiment, in which the length of the muscle may change, while the force it produces is 
constant. They are not obviously applicable if we wish to consider one of the most standard measures to define the character of a given muscle, its change of force as a function of time while muscle length is held constant (isometric contraction).

For the muscles of the larynx, there are few isotonic data. Almost all that we have for crossspecies comparison is isometric data, where there is no shortening of the muscle to provide an explicit cross-species scale in terms of muscle length for comparison. A comparison of the results of these experiments makes it clear that in general the homologous muscles of smaller organisms are faster, but it is not obvious how to devise a precise basis for cross-species comparisons.

First we must consider what physiological units may be appropriate for such comparison. A.V. Hill $^{17)}$ has described the twitch or simultaneous contraction of all of the motor units in a muscle, when the motor nerve is subjected to a supramaximal electrical stimulation, as "the elementary unit, the 'atom', of muscle activity" (1950). In the research of recent decades, many researchers have preferred other maneuvers to investigate muscle mechanics and muscle metabolism. However, it appears that the dimensions of twitch contractions can be scaled systematically across species.

Contraction time is the duration between the onset of isometric contraction and the peak of contractile force. However, there are no directly measured contraction time data for the human larynx. We have noted that some investigators have directly substituted values for dog for those of humans. It may be that a scaling strategy will be more precise in approaching this objective.

The larynx functions primarily in a respiratory context. The duration of the resting respiratory cycle is likely to be related to the contraction or laryngeal muscles which participate in it millions of times in a stereotyped manner, in activity which at least for PCA and TA starts in the womb (Mortola and Fisher ${ }^{20)}$ 1988) and usually ends only at death.

A very simple procedure might be to ask, what proportion of the duration of the resing breath cycle of that species is the contraction time of the muscle in question? The average duration of this cycle is available for most species for which we have measurements on laryngeal muscle mechanics.

Since we have the most published data for cricothyroid muscle contraction, from rat to sheep, these data were first plotted against the duration of the respiratory cycle in each species, based on adult

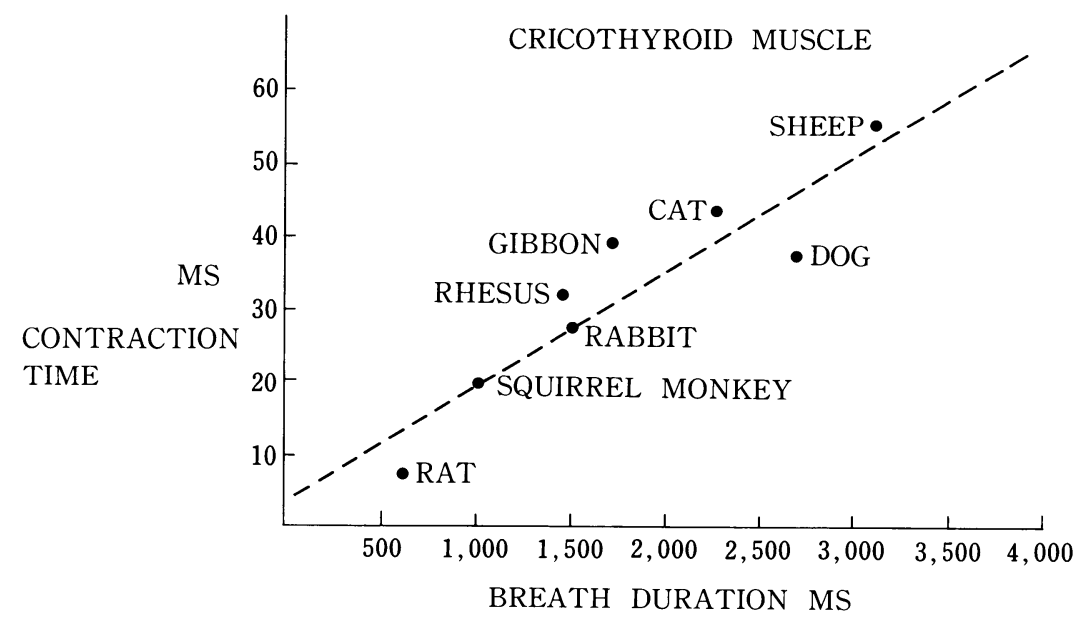

Fig. 1 Mean twitch contraction time in milliseconds (ms) for the cricothryoid muscle of indicated species (references in Cooper, Shindo, Hast, Sinha, and Rice, 1994) is plotted as a function of the mean duration of respiratory cycle for that species in seconds. Interrupted line is based on linear regression for which the mean of availiable data for each species is taken as a single value. 
values from standard tables, resulting in a respectable approximation to a straight line (Fig. 1). A complete listing of the data sources is given in Cooper, Shindo, Hast, et $\mathrm{al}^{21}$. (1994). The correlation coefficient for a linear regression between the average duration of the respiratory cycle of the species and the average contraction time for the published data is $r=.903$.

Then analogous plots were constructed for the other laryngeal muscles. The available data, although not as abundant for other muscles, did not contradict the hypothesis that the contraction time of each laryngeal muscle is a particular fraction of the duration of the respiratory cycle, constant across all of the mammalian species studied. we have seen that respiration duration is a power function of animal mass, so the link between laryngeal muscle contraction time and body mass can be taken as established. It is startling that data from animals which have undergone millions of years of divergent evolution demonstrate agreement in this regularity.

\section{Laryngeal Muscle Fatigue}

So far, the number of studies of fatigue resistance of laryngeal muscles is limited, although they would provide important information on laryngeal muscle metabolism (Cooper, Partridge, and Alipour-Haghighi $^{22)}$ 1993). Here our question is, can we use data from other animals to estimate the fatigue charcteristics of human laryngeal muscles?

Muscle fatigue may have several different physiological bases. For this reason, different procedures to test fatigue resistance may have different effects, which has lead to partial standardization of procedures. In general, these procedures measure the extent of decline of maximal twitch or tetanic contraction over time during a sequence of repeated contractions with fixed duration. Absolute force values can be eliminated, if the force is considered in terms of ratios, e.g. if we consider the fatigue decrement as the ratio of muscle contractile force after the fatigue procedure to the force of the initial contraction. For instance, Burke, Levine, Zajac, Tsairis and Engel $^{23)}$ (1971) assessed fatigue susceptibility of single motor units by using a two minute regime of stimulation at 40 pulses per second, in trains of stimulation each lasting $330 \mathrm{~ms}$, repeated one time per second. The quotient of the tension produced in the last train divided by that produced in the first train was termed the fatigue index, and has been used in a number of studies.

One such study has compared the resistance to fatigue of rat laryngeal muscles directly to that of human ones (Hinrichsen and Dulhunty, 1982) ${ }^{24)}$, The authors concluded from the rapid rate of fatigue of rat PCA muscle that human laryngal muscles would be fatiguable. This is puzzling, because in terms of its structural specialization for the delivery of oxygen to the fibers, PCA might be expected to be the most fatigue-resistant of laryngeal muscles (Cooper, Partridge and Alipour-Haghighi ${ }^{22)}$, 1993).

What is the problem? Is the rat in the in vitro preparation used here in some sense a poor model for the study of this question? Can some sort of specific scaling be applied to the dynamic aspects of these muscles which will permit more systematic interpretation of the results of such experiments?

It follows from the argument of the preceding section that the response to a fatigue maneuver would need to be scaled in terms of physiological time, in order to permit cross-species comparison. Hill $(1950)^{17}$ suggests that scaling of the time to fatigue in a given gesture is proportional to the linear difference in scale. Let us suppose that the linear dimensions of one animal are ten times as great, or it is 1000 times as heavy, as another animal. In that case, Hill ${ }^{17}$ ) suggest (1950), it will take ten times as long to become exhausted.

Assuming that this ratio applies to individual muscles, then based on animal mass, a Norway rat's laryngeal muscle would fatigue about four to six times more rapidly than the homologous human one, depending on whether McMahon's ${ }^{14)}$ (1980) or Hill' $\mathrm{S}^{25)}$ (1956) version of physiological time is used. Although greater precision is required as to how this temporal normalization should be carried out, cross-species comparison of time to fatigue without temporal normalization is likely to be misleading.

\section{Biological Scaling and Cell Size}

Cells of a given kind vary little in absolute size 
across species. A cervical motor neuron of a mouse has abont half the diameter of the same type of call in an elephant, although the linear dimensions of the elephant are at least fifty times greater (Thompson $\left.^{5)}, 1961\right)$. Why are the cell dimensions not linearly scaled up in large organisms? Hill $(1956)^{25)}$ suggested that this is a result of the limitations on the rate at which oxygen (and presumably other metabolic substances) can be supplied to the interior of the cell.

The result of this is that individual structures may contain many fewer units in small organisms than in large ones, and have a simpler structure in the small organisms. Thus, in smaller organisms, individual neurons of a given type are slightly smaller, and fewer of them compose a given structure. Nerves containing groups of related fibers, such as motor nerves, contain fewer motor fibers in small organisms, and homologous muscles also contain fewer fibers. The available data in this area are limited, so that in some cases it is necessary to make estimates. However, it will be useful to consider how variation of animal size will affect such estimates.

\section{Muscle Fiber Diameter}

First let us consider empirical data published by Kersing in his ${ }^{26)}$ (1983) Dutch dissertation on the histology and histochemistry of the muscle fibers of TA muscle fibers, studied in animals from mouse to man. This data set has the advantage that it relies on consistent histological procedures, so that shrinkage effects will be consistent. For other muscles, we shall rely especially on the study of seven different domestic ungulate and carnivore species by Mascarello and Veggetti ${ }^{27)}$ (1979), for the same reason.

From this data, it appears that the diameter of a given type of laryngeal muscle fiber varies little across smaller spccies, but increases progressively in animals with a mass from about thirty kilograms on up. However, this increase is far from proportional to the third root of mass. If so, the diameter of the horse's laryngeal muscle fibers would be about twelve times that of the rat, while it is about 1.5 times rat laryngeal muscle fiber diameter in fact.

Overlooking minor differences in the muscle con- figuration, the number of fibers in a given laryngeal muscle must gradually decrease with animal mass. Neural control is exercised over a much smaller numbers of fibers in a given laryngeal muscle of the mouse than in the homologous muscle in man.

\section{Motoneuron Diameter}

Now let us consider the scaling of the nervous system control over these muscles. A first question is the size of the brain stem nuclei involved. The mass of the brain varies approximately proportionally to the $2 / 3$ power of the animal's mass (Stahl ${ }^{3)}$ 1965). If the size of individual motoneurons in the nuclous ambiguus of the medulla controlling the larynx remains approximately the same across species, then their number is expected to decrease to with the mass of the brain.

Quantitative information on the size and number of the motor neurons of the nucleus ambiguus is limited, but some evidence suggests that there is an increase in the size of these motoneurons with animal mass (Hinrichsen and Ryan ${ }^{28)} 1981$; Lobera, et $\mathrm{al}^{29)} .1981$; Davis and Nail ${ }^{30)}, 1984$ ), Further information, especially from larger animals, is required to clarify this point (Gacek and Malmgren ${ }^{31}$, 1992). However, neuroanatomists are cautious about the effect of procedural artefacts on such measurements. Available findings suggest that the decrease in the number of laryngeal motoneurons in small animals is not as great as it would be if the motoneuron cell size were constant across species.

\section{Motor Unit Size}

A motor unit is defined as an alpha motoneuron and axon together with all the muscle fibers which it innervates by the terminal branches of its axon. The size of the motor units of a muscle is estimated by dividing the number of muscle fibers by the number of motor axons in the nerve which innervates it, or by the number of alpha motoneurons from which they originate. This is a mean quantity, since the number of muscle fibers constituting motor units varies within a given muscle. Small motor units are part of more precise control systems than large ones. Early estimates of the size of human laryngeal motor units ranged widely, from 
one to 247 muscle fibers (Faaborg-Andersen, $1957)^{15}$. Technical problems of several sorts may account for the disparity of the results.

In the study by English and Blevins $(1969)^{32}$, nerve and muscle fiber counts for some laryngeal muscles of man and cat were made. This study found a mean of 22.5 fibers per motor unit in three specimens of human cricothyroid on the assumption that two thirds of the innervating nerve fibers were motor. For cats, studies of the thyroarytenoid and lateral cricoarytenoid muscles also were made. There is rough agreement between the numbers of cat motor nerve fibers in the study of English and Blevins ${ }^{32)}$ (1969) and the number of motor neurons for individual cat laryngeal muscles observed in the study of Davis and Nail $(1984)^{30)}$. Thus it appears that in human cricothyroid muscle, there are over three times as many motor units as in cat, and about four times as many as in rat (Hinrichsen and Ryan $1982^{33)}$ ). Since there are half again as many muscle fibers in human cricothryoid as in cat's there are half as many muscle fiber in the average human cricothyroid motor unit as in cat. These findings are limited, and other quantitative data are required to make them more precise.

However, available findings suggest that the precision of lsryngeal motor control as quantified by the numbor of muscle fibers in motor unite increases with the mass of mammalian species. The number of muscle fibers in a muscle of a given species will be determined by fiber diameter and the number of fibers required to produce the force requisite in a larynx of a given size, scaled by muscle crosssectional area. More precise clarification is required for us to understand what determines the number of motor neurons controlling a given laryngeal muscle in each species. Without this information, crossspecies estimation of laryngeal motor unit size is not possible.

\section{Conclusion}

One approach to the integration of findings on the physiology of the larynk has been discussed. This is an approach which considers the larynx in its mammalian context. Analysis in terms of size of structures is not the only possible approach to such questions, but it is the approach which has been most systematically developed. More data are needed from large and small animals, gathered with uniform procedures, in order to provide a context which makes it possible to generalize about the function of the mammalian larynx, and to compare findings with or estimate human laryngeal function. We risk error if we substitute data from any other species directly for human values.

The author is grateful to Dr. K. Kitajima and members of the Japanese Society of Logopedics and Phoniatrics for the opportunity to address their 45th meeting. But a researcher also has many debts to those who make his work possible. This work was begun and largely carried out during a dozen years' association with the Departmer of Otolaryngolgy and Head and Neck Surgery of the University of Southern California, and its chairman, Dr. Dalc Rice. External support from the Voice Foundation and the National Institutes of Health has also supprted related research.

\section{Referencbs}

1) Hast, M.H. : The Primate Larynx. Acta Otolaryngol, $67:$ 84-92, 1969.

2) Desmond, A. Huxlev : From Devil's Disciple to Evolution's High Priest Reading, MA : Addison -Wesley, 1993.

3) Stahl, W. : Organ weights in primates and other mammals. Science, 150 : 1039-1042, 1965.

4) Stahl, W. Scaling of respiratory variables in mammals. J Appl Physiol, 22 : 453-460, 1967.

5) Thompson, D.W. : On Growth and Form et. JT Bonner Cambridge UK : Cambridge University Press, 1961.

6) Huxley, J.S. : Problems of Relative Growth New York : Dial Press. 1932.

7) McMahon, T.A., Bonner, J.T.: On Size and Life New York : Scientific American Book, 1983.

8) Peters, R.H. : The Ecological Implications of Body Size. Cambridge, UK : Cambridge University Press, 1983.

9) Schmidt-Nielsen, K. : Scaling Cambridge, UK : Cambridge University Press, 1984.

10) Calder, W.A. : Size, Function, and Life History Mineola, New York : Dover, 1996.

11) Brown, J.H., West, G.B. : Scaling in Biology 
Oxford University Press, 2000.

12) Batschelet, E. : Introduction to Mathematics for Life Scientists. Second ed. New York-Heidelberg-Berlin : Springer-Verlag, 1976.

13) Harrison, D.E.N. : The Anatomy and Physiology of the Mammalian Larynx Cambridge, UK : Cambridge University Press, 1995.

14) McMahon, T.A. : Scaling physiological time. pp. 131-163 in Lectures on Mathematics in the Life Sciences, vol. 13, 1980.

15) Faaborg-Andersen, K. : Electromyographic Investigation of Intrinsic Laryngeal Muscles in Humans. Acta Physiol Scand 41, suppl. : 140, 1957.

16) Wilkie, D.R. : Muscle Second cdition. London : Edward Arnold, 1976.

17) Hill, A.V. : "The Dimensions of Animals and their Muscular Dynamics” pp. 450-471 in Proceedings of the Roval Institution of Great Britain vol. XXXIV, Part III, No. 156, No. 4, 1949, 1950.

18) Hast, M.H. : Physiological Mechanisms of Phonation : Tension of the Vocal Fold Muscie Acta Otolaryngol, 62 : 309-318, 1966.

19) Cooper, D.S., Rice, D.H. : Contractile properties of canine thyroarytenoid muscle reinnervated from the ansa cervicalis. Ann Otol Rhinol Laryngol, 98(2) : 153-156, 1989.

20) Mortola, J.P., Fisher, J.T. : Upper Airway Reflexes in Newborns. pp. 303-357 in Respiratory Function of the Upper Airway edd. OP Mathew and G Sant' Ambrogio. New York -Basel : Marcal Dekker Inc. 1988.

21) Cooper, D.S., Shindo, S., Hast, M.H., et al. : Dynamic properties of the posterior cricoarytenoid muscle. Ann Otol Rhinol Laryngol 103(12) : 937-944, 1994.

22) Cooper, D.S., Partridge, L.D., Alipour -Haghighi, F. : Muscle energetics, vocal efficiency, and laryngeal biomechanics. pp. 37-92 in Vocal Fold Physiology : Frontiers in Basic Science ed. IR Titze. San Diego : Singular. 1993.

23) Burke, R.E., Levine, D.N., Zajac, F.E., et al. :
Mammalian motor units : physiologic-histochemical correlation in three types in cat gastrocnemius. Science 174 : 709-712, 1971.

24) Hinrichsen, C., Dulhunty, A. : The contractile properties, histochemistry, ultrastructure and elctrophysiology of the cricothryoid and posterior cricoarytenoid muscles in the rat. J Musc Res Cell Motil 3 : 169-190, 1982.

25) Hill, A.V. : The Design of Muscles British Medical Bulletin 12 : 165-166, 1956.

26) Kersing, W. : De Stembandmusculatuur. Utrecht : Drukerij Elinkwijk BV. 1983.

27) Mascarello, F., Veggetti, A. : A comparative histochemical study of intrinsic laryngeal muscles of ungulates and carnivores. Basic and Applied Histochemistry (Rivista di istochimica) 23 : 103-125, 1979.

28) Hinrichsen, C., Ryan, A.T. : Localization of laryngeal motoneurons in the rat. Experimental Neurology 74 : 341-355, 1981.

29) Lobera, B., Pasaro, R., Gonzales-Baron, S. et al. : A morphological study of ambiguus nucleus motoneurons innervating the laryngeal muscles in the rat and the cat. Neurosci Letters $23: 125$ $-130,1981$.

30) Davis, P.J., Nail, B.S. : On the Location and Size of Laryngeal Motoneurons in the Cat and Rabbit. J Comp Neurol 230 : 13-32, 1984.

31) Gacek, R., Malmgren, L. : "Laryngeal Motor Innervation-Central" pp. 29-35 in Neurologic Disorders of the Larynx A Blitzer, MF Brin, CT Sasaki, S Fahn, \& KS Harris. New York : Thieme. 1992.

32) English, D.T., Blevins, C.E. : Motor Units of Laryngeal Muscles. Arch Otolarvngol 89 : 120 $-784,1969$.

33) Hinrichsen, C., Ryan, A.T. : The size of motor units in laryngeal muscles of the rat. Experientia 38 : 360-361, 1982.

別刷請求先： $\mathbf{T} 520-2192$ 大津市瀬田月輪町 滋賀医科大学医学部耳鼻咽喉科学教室 北嶋和智 